\title{
Evolution of a collapsing and exploding Bose-Einstein condensate in different trap symmetries
}

\author{
Sadhan K. Adhikari \\ Instituto de Física Teórica, Universidade Estadual Paulista, 01.405-900 São Paulo, São Paulo, Brazil
}

(Received 27 October 2004; published 12 May 2005)

\begin{abstract}
Based on the time-dependent Gross-Pitaevskii equation we study the evolution of a collapsing and exploding Bose-Einstein condensate in different trap symmetries to see the effect of confinement on collapse and subsequent explosion, which can be verified in future experiments. We make a prediction for the evolution of the shape of the condensate and the number of atoms in it for different trap symmetries (cigar to pancake) as well as in the presence of an optical lattice potential. We also make a prediction for the jet formation in different cases when the collapse is suddenly terminated by changing the scattering length to zero via a Feshbach resonance. In addition to the usual global collapse to the center of the condensate, in the presence of an optical-lattice potential one could also have in certain cases independent collapse of parts of the condensate to local centers, which could be verified in experiments.
\end{abstract}

DOI: 10.1103/PhysRevA.71.053603

PACS number(s): $03.75 . \mathrm{Nt}$

\section{INTRODUCTION}

Since the detection and study of Bose-Einstein condensates (BECs) of ${ }^{7} \mathrm{Li}$ atoms with attractive interaction [1], such condensates have been used in the study of solitons [2] and collapse [3]. In general an attractive condensate with number of atoms $N$ larger than a critical value $N_{\text {cr }}$ is not dynamically stable [1]. However, if such a strongly attractive condensate is "prepared" or somehow made to exist it experiences a dramatic collapse and explodes emitting atoms. The first demonstration of such a collapse was made with a ${ }^{7} \mathrm{Li}$ condensate by slowly increasing the number of atoms in it from an external source, while the BEC showed a sequence of collapse with the number of atoms $N$ oscillating around $N_{\text {cr }}[1]$. Such a collapse is driven by a stochastic process.

A dynamical study of a much stronger and violent collapse has been performed by Donley et al. [3] on an attractive ${ }^{85} \mathrm{Rb} \mathrm{BEC}$ [4] in an axially symmetric trap, where they manipulated the interatomic interaction by changing the external magnetic field exploiting a nearby Feshbach resonance [5]. In the vicinity of a Feshbach resonance the atomic scattering length $a$ can be varied over a huge range by adjusting an external magnetic field. Consequently, they changed the sign of the scattering length, thus transforming a repulsive condensate of ${ }^{85} \mathrm{Rb}$ atoms into an attractive one which naturally evolves into a collapsing and exploding condensate. Donley et al. provided a quantitative estimate of the explosion of this BEC by measuring different properties of the exploding condensate.

It has been realized that many features of the experiment by Donley et al. [3] on the collapsing condensate can be described [6-18] by the mean-field Gross-Pitaevskii (GP) equation [19]. To account for the loss of atoms from the strongly attractive collapsing condensate an absorptive nonlinear three-body recombination term is included in the GP equation [6]. However, we are fully aware that there are features of this experiment which are expected to be beyond mean-field description. Among these are the distribution of number and energy of emitted high-energy $\left(\sim 10^{-7}\right.$ Kelvin $)$ burst atoms reported in the experiment. Although there have been some attempts [9-11] to describe the burst atoms using the mean-field GP equation, now there seems to be a consensus that they cannot be described adequately and satisfactorily using a mean-field approach [13-15]. Also, the GP equation did not successfully predict the "time to collapse" (or the time lag to start the collapse after changing the sign of the scattering length) in all cases investigated in the experiment, as has been pointed out in Refs. $[12,16]$. However, the reason for the failure to predict the time to collapse is not clear.

The GP equation is supposed to deal with the zero- or very low-energy condensed phase of atoms and has been used to predict the time to collapse, evolution of the collapsing condensate including the very low-energy ( $\sim$ nano Kelvin) jet formation [3] when the collapse is suddenly stopped before completion by jumping the scattering length to $a_{\text {quench }}=0$ (noninteracting atoms) or positive (repulsive atoms) values. The jet atoms are slowly formed in the radial direction when the collapse is stopped in this fashion. In the experiment usually $a_{\text {quench }}=0$. It is emphasized that unlike the emitted uncondensed "hotter" missing and burst atoms reported in the experiment [3] the jet atoms form a part of the surviving "colder" condensate and hence should be describable by the mean-field GP equation. Saito et al. [9], Bao et al. [15] and this author [17] presented a mean-field description of jet formation and Calzetta et al. [14] treated jet formation exclusively as a quantum effect. The GP equation has also been used to study the conditions of stability as well as collapse [20] of a coupled condensate. More recently, the present author used successfully the GP equation to describe the essentials of the collapse dynamics of a mixture of a boson and fermion condensates [21].

In this paper we extend the study of the evolution of the collapsing and exploding condensate in different symmetries to see the effect of confinement on collapse and subsequent explosion. Future experiments may verify these predictions and thus provide a more stringent test for the mean-field GP equation. The experiment of Donley et al. was performed for an axially-symmetric cigar-shaped BEC. In the present analysis we extend our study to a spherical as well as an axially-symmetric pancake-shaped BEC. 
Lately, the periodic optical-lattice potential has played an essential role in many theoretical and experimental studies of Bose-Einstein condensation, e.g., in the study of Josephson oscillation [22] and its disruption [23], interference of matter wave [24], BEC dynamics on periodic trap [25], etc. The optical-lattice confinement creates a BEC in an entirely different shape and trapping condition form a conventional harmonic oscillator trapping. Consequently, one could have a collapse of a different nature in the presence of an opticallattice potential. We shall see in our study that under certain conditions of trap symmetry, in addition to the usual global collapse to the center, in the presence of the optical-lattice potential one could have independent local collapse of pieces of the condensate to local centers. In view of this we study the dynamics of a collapsing and exploding BEC of different symmetries prepared on a periodic optical-lattice potential. We study the evolution of the shape and size of the condensate as well as the jet formation upon stopping the collapse by making the BEC repulsive or noninteracting.

In Sec. II we present our mean-field model. In Sec. III we present our results that we compare with the experiment and other numerical studies. In Sec. III we also present a physical discussion of our findings and some concluding remarks are given in Sec. IV.

\section{NONLINEAR GROSS-PITAEVSKII EQUATION}

The time-dependent Bose-Einstein condensate wave function $\Psi(\mathbf{r} ; \tau)$ at position $\mathbf{r}$ and time $\tau$ allowing for atomic loss may be described by the following mean-field nonlinear GP equation [19],

$$
\begin{aligned}
& {\left[-i \hbar \frac{\partial}{\partial \tau}-\frac{\hbar^{2} \nabla^{2}}{2 m}+V(\mathbf{r})+g N|\Psi(\mathbf{r} ; \tau)|^{2}-\frac{i \hbar}{2}\left[K_{2} N|\Psi(\mathbf{r} ; \tau)|^{2}\right.\right.} \\
& \left.\left.+K_{3} N^{2}|\Psi(\mathbf{r} ; \tau)|^{4}\right]\right] \Psi(\mathbf{r} ; \tau)=0 .
\end{aligned}
$$

Here $m$ is the mass and $N$ the number of atoms in the condensate, $g=4 \pi \hbar^{2} a / m$ the strength of interatomic interaction, with $a$ the atomic scattering length. The terms $K_{2}$ and $K_{3}$ denote two-body dipolar and three-body recombination lossrate coefficients, respectively, and include the Bose statistical factors $1 / 2$ ! and $1 / 3$ ! needed to describe the condensate. The trap potential with cylindrical symmetry may be written as $V(\mathbf{r})=\frac{1}{2} m \omega^{2}\left(\rho^{2}+\nu^{2} z^{2}\right)+V_{\text {op }}$, where $\omega$ is the angular frequency in the radial direction $r$ and $\nu \omega$ that in the axial direction $z$ of the harmonic trap. The cigar-shaped condensate corresponds to $\nu<1$ and pancake-shaped condensate corresponds to $\nu>1$. The periodic optical-lattice potential in the axial $z$ direction created by a standing-wave laser field of wavelength $\lambda$ is given by $V_{\mathrm{op}}=\kappa E_{R} \cos ^{2}\left(k_{L} z\right)$ with $E_{R}$ $=\hbar^{2} k_{L}^{2} /(2 m), k_{L}=2 \pi / \lambda$ and $\kappa$ the strength. The normalization condition of the wave function is $\int d \mathbf{r}|\Psi(\mathbf{r} ; \tau)|^{2}=1$. Here we simulate the atom loss via the most important quintic three-body term $K_{3}$ [6-9]. The contribution of the cubic twobody loss term $K_{2}$ [26] is expected to be negligible [6,9] compared to the three-body term in the present problem of the collapsed condensate with large density and will not be considered here.
In the absence of angular momentum the wave function has the form $\Psi(\mathbf{r} ; \tau)=\psi(\rho, z ; \tau)$. Now transforming to dimensionless variables defined by $x=\sqrt{2} \rho / l, y=\sqrt{2} z / l, t=\tau \omega, l$ $\equiv \sqrt{\hbar /(m \omega)}$, and

$$
\phi(x, y ; t) \equiv \frac{\varphi(x, y ; t)}{x}=\sqrt{\frac{l^{3}}{\sqrt{8}}} \psi(\rho, z ; \tau),
$$

we get

$$
\begin{aligned}
& {\left[-i \frac{\partial}{\partial t}-\frac{\partial^{2}}{\partial x^{2}}+\frac{1}{x} \frac{\partial}{\partial x}-\frac{\partial^{2}}{\partial y^{2}}+\frac{1}{4}\left(x^{2}+\nu^{2} y^{2}-\frac{4}{x^{2}}\right)\right.} \\
& +\kappa \frac{4 \pi^{2}}{\lambda_{0}^{2}} \cos ^{2}\left(\frac{2 \pi}{\lambda_{0}} y\right)+8 \sqrt{2} \pi n\left|\frac{\varphi(x, y ; t)}{x}\right|^{2} \\
& \left.-i \zeta n^{2}\left|\frac{\varphi(x, y ; t)}{x}\right|^{4}\right] \varphi(x, y ; t)=0,
\end{aligned}
$$

where $n=N a / l, \lambda_{0}=\sqrt{2} \lambda / l$ and $\zeta=4 K_{3} /\left(a^{2} l^{4} \omega\right)$. The normalization condition of the wave function becomes

$$
\mathcal{N}_{\text {norm }} \equiv 2 \pi \int_{0}^{\infty} d x \int_{-\infty}^{\infty} d y|\varphi(x, y ; t)|^{2} x^{-1} .
$$

For $\zeta=K_{3}=0, \mathcal{N}_{\text {norm }}=1$, however, in the presence of loss $K_{3}>0, \mathcal{N}_{\text {norm }}<1$. The number of remaining atoms $N$ in the condensate is given by $N=N_{0} \mathcal{N}_{\text {norm }}$, where $N_{0}$ is the initial number of atoms.

In this study the term $K_{3}$ or $\zeta=4 K_{3}\left(a^{2} l^{4} \omega\right)$ will be used for a description of atom loss in the case of attractive interaction. The choice of $K_{3}$ has a huge effect on some experimental observables and the fact that it is experimentally not precisely determined is a problem for existing theory on the experiment. As in our previous study [17] we employ $\zeta=2$ and $K_{3} \sim a^{2}$ throughout this study. It was found [17] that this value of $\zeta(=2)$ reproduced the time evolution of the condensate in the experiment of Donley et al. [3] satisfactorily for a wide range of variation of an initial number of atoms and scattering lengths [7]. The present value $\zeta=2$ with $K_{3}$ $=\zeta a^{2} l^{4} \omega / 4$ leads to $[7,8] \quad K_{3} \simeq 8 \times 10^{-25} \mathrm{~cm}^{6} / \mathrm{s}$ at $a=$ $-340 a_{0}$ and $K_{3} \simeq 6 \times 10^{-27} \mathrm{~cm}^{6} / \mathrm{s}$ at $a=-30 a_{0}$, where $a_{0}$ is the Bohr radius. The experimental value of the loss rate is [26] $K_{3} \simeq 7 \times 10^{-25} \mathrm{~cm}^{6} / \mathrm{s}$ at $a=-340 a_{0}$ which is very close to the present choice. Of the theoretical studies, the $K_{3}$ values used by Santos et al. [11] $\left(K_{3} \simeq 7 \times 10^{-25} \mathrm{~cm}^{6} / \mathrm{s}\right.$ at $a$ $\left.=-340 a_{0}\right)$, Savage et al. $[12]\left(K_{3} \simeq 19 \times 10^{-27} \mathrm{~cm}^{6} / \mathrm{s}\right.$ at $a$ $\left.=-30 a_{0}\right)$, Bao et al. [15] $\left(K_{3} \simeq 6.75 \times 10^{-27} \mathrm{~cm}^{6} / \mathrm{s}\right.$ at $a$ $=-30 a_{0}$ ) and the present author [7] are consistent with each other and describes well the decay of the collapsing condensate. However, it seems unlikely that the much smaller value of $K_{3}\left(K_{3}=2 \times 10^{-28} \mathrm{~cm}^{6} / \mathrm{s}\right.$ at $\left.a=-30 a_{0}\right)$ used in Refs. [9,10] will properly describe the decay of the collapsing condensate.

\section{NUMERICAL RESULT}

We solve the GP equation (2.3) numerically using a timeiteration method based on the Crank-Nicholson discretization scheme elaborated on in Ref. [27]. We discretize the GP 


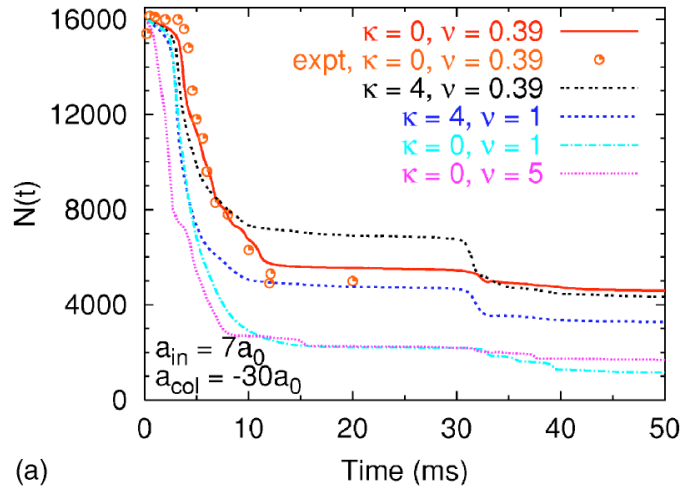

(a)

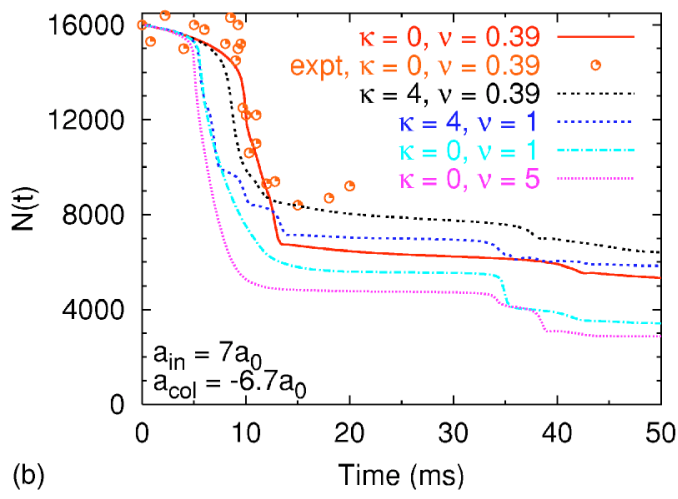

FIG. 1. (Color online) Number of remaining atoms $N(t)$ in the condensate of $16000{ }^{85} \mathrm{Rb}$ atoms after ramping the scattering length from $a_{\text {in }}=7 a_{0}$ to (a) $a_{\mathrm{col}}=-30 a_{0}$ and (b) $a_{\mathrm{col}}=-6.7 a_{0}$ as a function of evolution time in milliseconds. The unpublished and unanalyzed experimental points of Donley et al. [3] for $a_{\mathrm{col}}$ $=-6.7 a_{0}$ are taken from Bao et al. [15]. The curves are labeled by their respective optical lattice strength $\kappa$ and axial trap parameter $\nu$.

equation using time step $\Delta=0.001$ and space step 0.1 for both $x$ and $y$ spanning $x$ from 0 to 15 and $y$ from -30 to 30 . This domain of space was sufficient to encompass the whole condensate wave function in this study.

First, the numerical simulation is performed with the actual parameters of the experiment by Donley et al. [3], e.g., the initial number of atoms, scattering lengths, etc. Throughout this investigation we take the harmonic oscillator length $l=2607 \mathrm{~nm}$ and one unit of time $t=0.009095 \mathrm{~s}$ [7] consistent with the experiment of Donley et al. [3]. When we include an optical-lattice potential, the optical-lattice strength $\kappa$ is taken to be 4 , and the reduced wavelength $\lambda_{0}$ is taken to be 1 throughout this study. These optical-lattice parameters are consistent with the experiment by Cataliotti et al. $[22,23]$. The numerical simulation using Eq. (2.3) with a nonzero $\zeta(=2)$ immediately yields the remaining number of atoms in the condensate after the jump in scattering length.

\section{A. Evolution of the number of atoms in the condensate}

In the experiment the initial scattering length $a_{\text {in }}(>0)$ of a repulsive condensate is suddenly jumped to $a_{\text {col }}(<0)$ to start the collapse. The remaining number $N(t)$ of atoms versus time for an initial number of atoms $N_{0}=16000$ and an initial scattering length $a_{\mathrm{in}}=7 a_{0}$ are shown in Figs. 1(a) and 1(b) for final scattering lengths after collapse $a_{\mathrm{col}}=-30 a_{0}$ and $-6.7 a_{0}$, respectively. In both cases the experimental data for $\kappa=0$ and $\nu=0.39$ (cigar-shaped condensate) are in agreement with the theoretical simulation without any adjustable parameter. For $a_{\mathrm{col}}=-6.7 a_{0}$, the unpublished experimental data of [3] as shown in Fig. 1(b) are as quoted in Bao et al. [15]. These data are not fully analyzed and for large time are expected to be bigger than the actual number of atoms. This is due to the difficulty in separating the remnant condensate from the oscillating atom cloud surrounding it [3]. In addition, in Figs. 1 we plot the results for $\kappa=4$ and $\nu=0.39$ (cigar-shaped condensate with optical-lattice potential); $\kappa=4$ and $\nu=1$ (spherical condensate with optical-lattice potential); $\kappa=0$ and $\nu=1$ (spherical condensate); and $\kappa=0$ and $\nu=5$ (pancake-shaped condensate).

As the repulsive condensate is quickly turned attractive at $t=0$, via a Feshbach resonance, the condensate starts to collapse and once the central density increases sufficiently it loses a significant portion of atoms in an explosive fashion via three-body recombination to form a remnant condensate in about $15 \mathrm{~ms}$ as can be seen in Figs. 1. After explosion the number of atoms in the remnant continues to be much larger than the critical number of atoms $N_{\mathrm{cr}}$ and it keeps on losing atoms at a much slower rate without undergoing a violent explosion. However, in some cases the remnant undergoes a smaller secondary explosion while it loses a reasonable fraction of atoms in a small interval of time. This happens when the number of atoms in the remnant is much larger than $N_{\text {cr }}$ so as to initiate a secondary collapse and explosion. Prominent secondary explosions in the presence of optical-lattice potential are found in different cases in Figs. 1 for $40>t$ $>30$.

\section{B. Time to collapse}

Another important aspect of collapse is the "time to collapse" or the time to initiate the collapse and explosion $t_{\text {collapse }}$ after the repulsive condensate is suddenly made attractive at $t=0$. Collapse is characterized by a sudden rapid emission of atoms from the condensate. From Figs. 1 we find that the time to collapse is the shortest for a pancake-shaped symmetry $(\nu>1)$ and is the longest for a cigar-shaped symmetry $(\nu<1)$. The inclusion of an optical-lattice potential has no effect on the time to collapse for a spherical or pancake-shaped symmetry. However, its inclusion reduces the time to collapse for a cigar-shaped symmetry. These features of the time to collapse are illustrated in Fig. 2 where we plot $t_{\text {collapse }}$ vs $\left|a_{\text {collapse }}\right| / a_{0}$ of the collapse of a condensate of 6000 atoms originally in a noninteracting state with scattering length $a_{\text {initial }}=0$. Then suddenly its scattering length is changed to a negative (attractive) value $a_{\text {collapse }}$ and its $t_{\text {collapse }}$ is obtained. Donley et al. experimentally measured $t_{\text {collapse in }}$ this case for $\nu=0.39$ and $\kappa=0$ and here we provide the same for other values of trap symmetry $\nu$ and also in the presence of a optical lattice potential with $\kappa=4$. It should be recalled that the prediction of the GP equation by this author and others $[9,12,16]$ does not very well describe the experimental results of Donley et al. for the time to collapse. The inclusion of the optical-lattice potential has reduced the time to collapse in a cigar-shaped condensate $(\nu=0.1)$. 


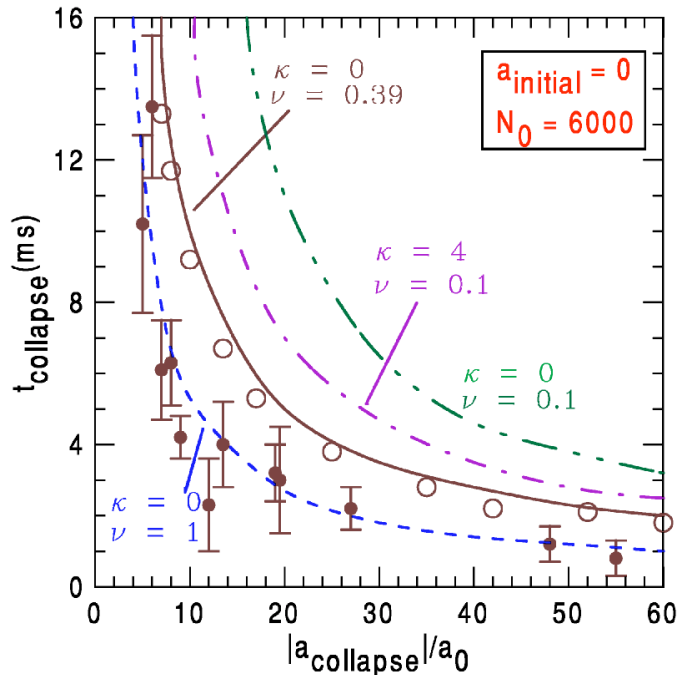

FIG. 2. (Color online) The time to collapse $t_{\text {collapse }}$ vs $\left|a_{\text {collapse }}\right| / a_{0}$ for $a_{\text {initial }}=0, N_{0}=6000$. Solid circle with error bar: experiment [3] with $\nu=0.39$ and $\kappa=0$; open circle: mean-field model of [9] with $\nu=0.39$ and $\kappa=0$; full line: the present result with $\nu$ $=0.39$ and $\kappa=0$; dashed line: the present result with $\nu=1$ and $\kappa$ $=0$; dashed-dotted line: the present result with $\nu=0.1$ and $\kappa=4$; and dashed-doubled-dotted line: the present result with $\nu=0.1$ and $\kappa$ $=0$.

The above features of time to collapse could be understood on a physical ground. In a cigar-shaped condensate the average distance among the atoms is larger than that in a pancake-shaped condensate of the same volume. Hence, due to atomic attraction a cigar-shaped condensate has to contract during a larger interval of time than a pancake-shaped condensate before the central density increases sufficiently to start an explosion. This justifies a larger time to collapse for a cigar-shaped condensate. In the presence of an opticallattice potential for cigar-shaped symmetry the optical-lattice divides the condensate in a large number of pieces. What predominates in the collapse of such a condensate is the collapse of an individual piece to a local center rather than to the global center of the condensate via tunneling. This is a quicker process than the collapse of the whole condensate to the global center. This is why the time to collapse is shorter for a cigar-shaped condensate in an optical-lattice trap than a cigar-shaped condensate in a harmonic trap alone. In a pancake-shaped symmetry the number of optical-lattice sites inside the condensate is small. In this case a separation of the condensate in a smaller number of pieces does not aid in the collapse, as the different slices of the condensate has to collapse essentially towards the center of the condensate before the explosion starts. Hence the optical-lattice potential has almost no effect on the time to collapse in the pancakeshaped or spherical symmetry.

Another aspect of Figs. 1 worth mentioning is that the number of atoms in the remnant condensate after the first explosion is larger in the presence of an optical-lattice potential. Due to optical-lattice barriers one essentially has local collapse of different pieces of the condensate in this case as opposed to a global collapse to the center of the condensate in the case of a harmonic trap alone. Consequently, the col-
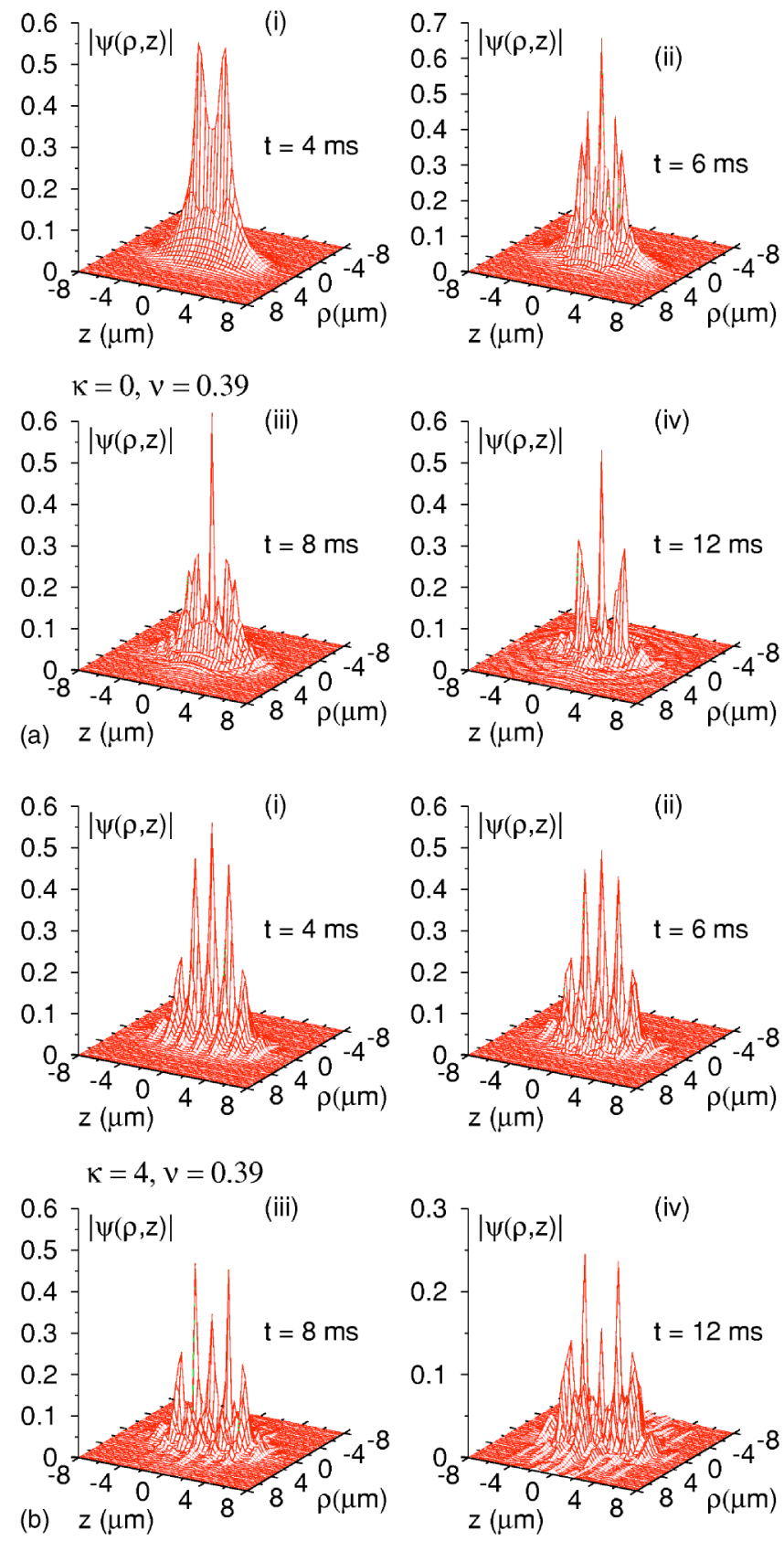

FIG. 3. A view of the evolution of the residual condensate wave function $|\psi(\rho, z)|$ in arbitrary units for initial scattering length $a_{\text {in }}$ $=7 a_{0}$, final scattering length $a_{\mathrm{col}}=-30 a_{0}$, initial number of atoms $N_{0}=16000$ at times $t=$ (i) $4 \mathrm{~ms}$, (ii) $6 \mathrm{~ms}$, (iii) $8 \mathrm{~ms}$ and (iv) $12 \mathrm{~ms}$ for (a) $\kappa=0, \nu=0.39$ and (b) $\kappa=4, \nu=0.39$.

lapse is more violent with greater loss of atoms in the absence of an optical-lattice trap. This is why the remnant number after the first explosion is larger in the presence of an optical trap.

\section{Evolution of the shape of the condensate}

Next we consider the evolution of the shape of the residual condensate. In Fig. 3(a) we show the profile of the wave function $\psi(\rho, z)$ at different times during explosion for 

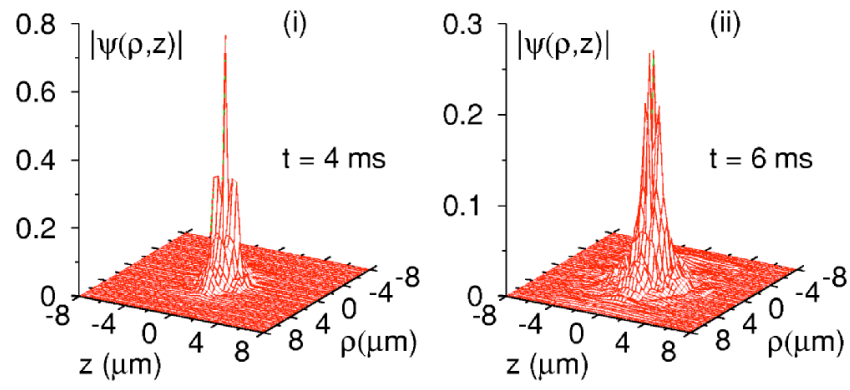

$$
\kappa=0, v=5
$$
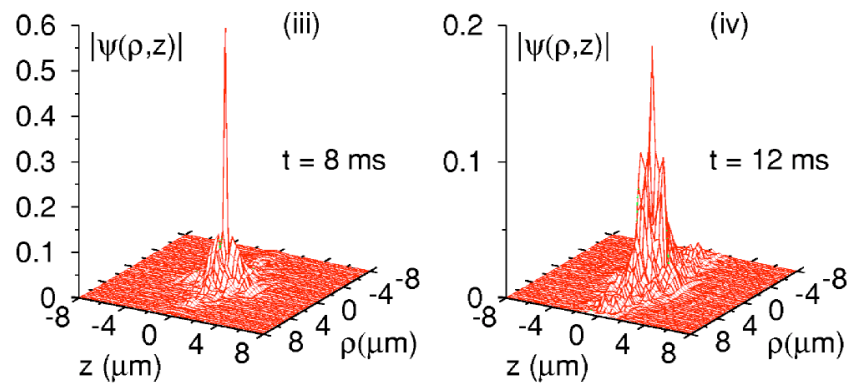

FIG. 4. Same as in Figs. 3 for $\kappa=0, \nu=5$.

$N_{0}=16000, \kappa=0, \nu=0.39, a_{\text {in }}=7 a_{0}$, and $a_{\text {col }}=-30 a_{0}$. This is the case of a cigar-shaped condensate used in the experiment [3]. During explosion the condensate wave function develops a three-peak structure noted before in [9]. In Fig. 3(b) we illustrate the profile of the wave function $|\psi(\rho, z)|$ at different times during explosion of the condensate formed in an optical-lattice potential with $\kappa=4$ in addition to the axial harmonic trap: the other parameters remaining the same as in Fig. 3(a). The condensate now develops a distinct multi-peak structure along the optical lattice in place of the three-peak structure in the absence of the optical-lattice potential. However, the number of peaks in the wave function is less by a factor of two to three than the number of pits of the opticallattice potential. The number of distinct peaks in the wave function in this case is five as can be seen in Fig. 3(b).

The above distinct peaks in the wave function in the presence of the optical-lattice potential may have an interesting application in the generation of radially bound and axially free bright solitons. The wave function of Fig. 3(b) is axially bound. However, if the axial trap and the optical-lattice potential are removed, or better an expulsive potential is applied in the axial direction, the wave function will expand axially. The side peaks of the wave function can evolve into separate solitons and come out in the axial direction which can be used as bright solitons in other experiments.

The scenario of the evolution of the condensate is entirely different for pancake-shaped condensate with $\nu>1$. In that case the condensate is squeezed in the axial direction and a single peak, rather than multiple peaks, is formed in the condensate wave function. This is illustrated in Fig. 4 where we plot the condensate wave function for $\kappa=0$ and $\nu=5$, the other parameters of simulation being the same as in Figs. 2. The use of optical-lattice potential in this case also does not lead to prominent peaks in the wave function in the axial direction.
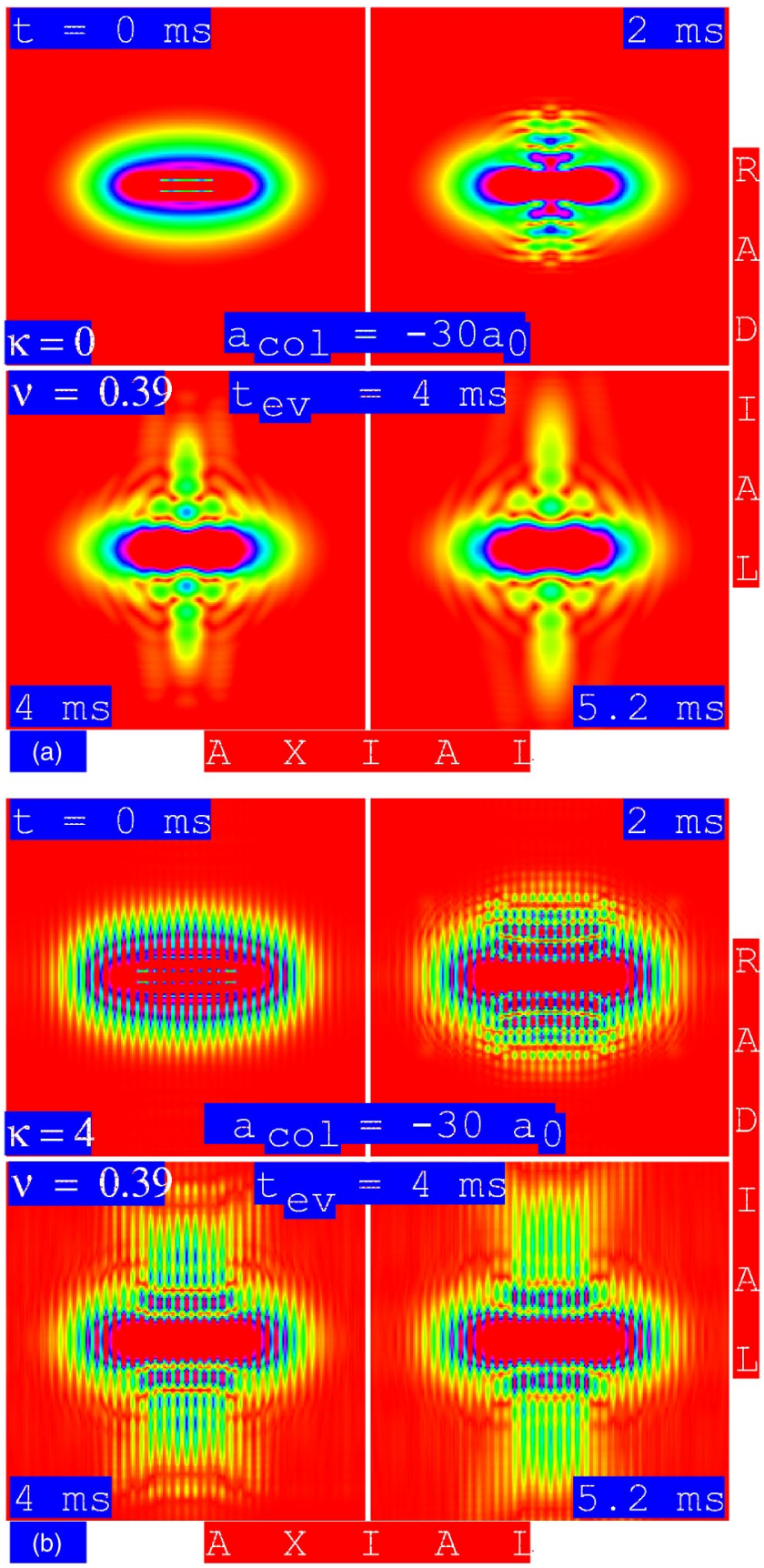

FIG. 5. (Color online) A view of the evolution of radial jet at times $t=0,2 \mathrm{~ms}, 4 \mathrm{~ms}$ and $5.2 \mathrm{~ms}$ on a mat of size $16 \mu \mathrm{m}$ $\times 16 \mu \mathrm{m}$ from a contour plot of $|\psi(\rho, z)|$ for initial scattering length $a_{\mathrm{in}}=7 a_{0}$, final scattering length $a_{\mathrm{col}}=-30 a_{0}$, initial number of atoms $N_{0}=16000$, (a) without an optical-lattice potential $(\kappa=0)$ and (b) with an optical-lattice potential $(\kappa=4)$. In both cases the jet formation was started by jumping the scattering length to $a_{\text {quench }}=0$ after a time $t_{\mathrm{ev}}=4 \mathrm{~ms}$ of the beginning of collapse.

\section{Jet formation}

Another interesting feature of the experiment of Donley et al. [3] is the formation of a jet. As the collapse was suddenly terminated after an evolution time $t_{\mathrm{ev}}$ by jumping the scattering length from $a_{\text {col }}$ to $a_{\text {quench }} \geqslant 0$, the jet atoms were slowly formed in the radial direction. In the strongly collapsing con- 

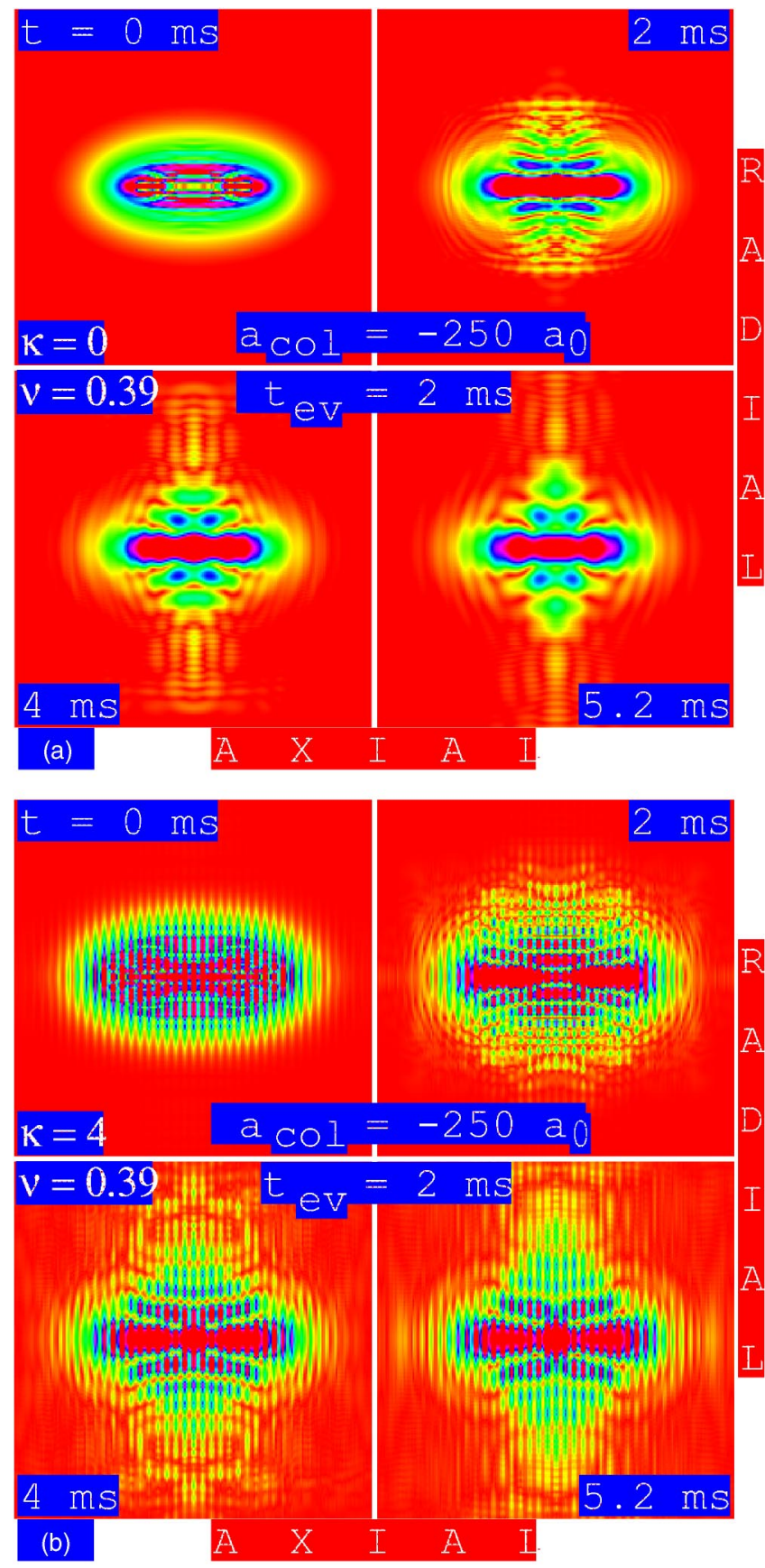

FIG. 6. (Color online) Same as in Fig. 5 for $a_{\mathrm{col}}=-250 a_{0}$, and $t_{\mathrm{ev}}=2 \mathrm{~ms}$.

densate, local radial spikes are formed during particle loss as can be seen from a plot of the numerically calculated wave function [7] and in experiment [3]. During particle loss the top of the spikes are torn and ejected out and new spikes are formed until the explosion and particle loss are over. There is a balance between central atomic attractive force and the outward kinetic pressure. If the attractive force is now suddenly removed by stopping the collapse by applying $a_{\text {quench }}$ $=0$, the highly collapsed condensate expands due to kinetic pressure, becomes larger and the recombination of atoms is greatly reduced. Consequently, the spikes expand and develop into a prominent jet [3].

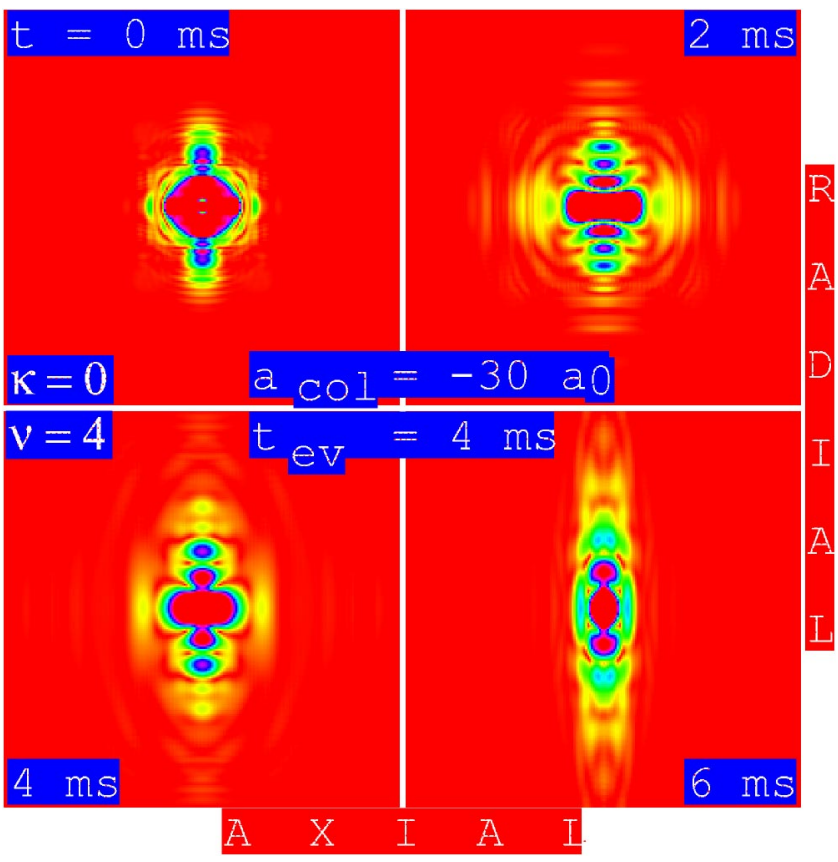

FIG. 7. (Color online) Same as in Fig. 5(a) for $\nu=4$ and $t=0$, $2 \mathrm{~ms}, 4 \mathrm{~ms}$ and $6 \mathrm{~ms}$.

Now we consider the jet formation as in the experiment of Donley et al. [3] at different times $t$ of the collapsing condensate after jumping the scattering length suddenly from $a_{\mathrm{col}}=-30 a_{0}$ to $a_{\text {quench }}=0$ during explosion at time $t_{\mathrm{ev}}$ from the beginning of collapse and explosion. The initial scattering length $a_{\text {in }}=7 a_{0}$ and number of atoms $N_{0}=16000$. In Figs. 5(a) and 5(b) we show the contour plot of the condensate for $t_{\mathrm{ev}}=2 \mathrm{~ms}$, without $(\kappa=0)$ and with $(\kappa=4)$ an optical-lattice potential, respectively, at different times $t=0,2 \mathrm{~ms}, 4 \mathrm{~ms}$, and $5.2 \mathrm{~ms}$ after jumping the scattering length to $a_{\text {quench }}=0$. A prominent radial jet is formed slowly at time $t=4-6 \mathrm{~ms}$ after stopping the collapse at $t_{\mathrm{ev}}=4 \mathrm{~ms}$. The jet is much less pronounced for $t_{\mathrm{ev}}=2,8$ and $10 \mathrm{~ms}$ (not shown in the figure) compared to the jet in Figs. 5. There is a fundamental difference between the jets in Figs. 5(a) and 5(b) in the absence and presence of the optical-lattice potential. In Fig. 5(a) the absence of the optical potential the jet is narrow, whereas in Fig. 5(b) it is wide and spread over a number of optical lattice sites.

In addition, we studied jet formation for different values of $a_{\mathrm{col}}$ in place of $a_{\mathrm{col}}=-30 a_{0}$ and find that the general scenario remains similar. For example, for $a_{\mathrm{col}}=-250 a_{0}$, the collapse and subsequent explosion starts at a small time. So for a good formation of the jet a smaller value of $t_{\mathrm{ev}}$ is to be preferred. In Figs. 6 we show the jet formation for $a_{\text {col }}$ $=-250 a_{0}$ and $t_{\mathrm{ev}}=2 \mathrm{~ms}$. In this case the shape of the jet is different from that in Figs. 5. However, as in Figs. 5, the jet gets broadened in the presence of the optical-lattice potential.

Next we study the effect of the axial trap symmetry on jet formation. In Figs. 5 and 6 the harmonic trap has cigar symmetry $(\nu=0.39<1)$. If it is changed to pancake symmetry $(\nu>1)$, the condensate and the jet gets compressed in the axial direction. Consequently, the radial jet is not very pronounced and cannot be clearly distinguished from the con- 
densate. This is illustrated in Fig. 7 for a pancake-shaped trap with $\nu=4$ in the absence of an optical-lattice potential. In this case at $t=6 \mathrm{~ms}$ the condensate is more extended in the radial direction compared to the condensate at $t=0$ due to the formation of a jet. However, due to the overall compression of the condensate in the axial direction the jet cannot be easily separated from the condensate. In contrast, in Figs. 5 the jet is easily separated from the central condensate. So pancake symmetry is not ideal for the study of a jet. The situation does not change in the presence of an optical-lattice potential superposed on a pancake-shaped trap.

\section{CONCLUSION}

In conclusion, we have employed a numerical simulation based on the accurate solution [27] of the mean-field GrossPitaevskii equation with a cylindrical trap to study the evolution of a collapsing and exploding condensate as in the experiment of Donley et al. [3]. In the GP equation we include a quintic three-body nonlinear recombination loss term [6] that accounts for the decay of the strongly attractive condensate. We also extend our investigation to different trap symmetries and including an optical-lattice potential in the axial direction. In addition to studying the evolution of the size and the shape of the condensate, we also study the jet formation as observed experimentally. Without any adjustable parameter the result of the present and previous simula- tions of this author are in good agreement with some aspects of the experiment by Donley et al. [3].

It is interesting to emphasize that the GP equation does describe some but not all aspects of the collapse experiment by Donley et al. and its predictions for the "time to collapse" do not agree well with experiment $[12,16]$. The failure to explain the time to collapse is dramatic as intuitively one should expect the mean-field model to be a faithful model for time to collapse involving the dynamics of the coldest atoms in the condensate. However, there are aspects of experiments which cannot be described by mean-field models $[13,14,16,17]$, e.g., the dynamics of missing and burst atoms [3]. Furthermore all numerical studies of this experiment suffer from a limited knowledge of the three-body loss rate $K_{3}$, and even though many experimental features can be described by a suitable choice of $K_{3}$, no value of $K_{3}$ yields simultaneous agreement between predictions of the GP equation and all observable quantities of the experiment. In this situation it would be of great theoretical and experimental interests to see if a repeated experiment with different trap parameters (and also including an optical-lattice potential) would help to understand the underlying physics and could make use of numerical studies such as those described in the present paper.

\section{ACKNOWLEDGMENTS}

The work is supported in part by the CNPq of Brazil.
[1] J. M. Gerton, D. Strekalov, I. Prodan, and R. G. Hulet, Nature (London) 408, 692 (2001); C. C. Bradley, C. A. Sackett, J. J. Tollett, and R. G. Hulet, Phys. Rev. Lett. 75, 1687 (1995); C. C. Bradley, C. A. Sackett, and R. G. Hulet, ibid. 78, 985 (1997).

[2] L. Khaykovich, F. Schreck, G. Ferrari, T. Bourdel, J. Cubizolles, L. D. Carr, Y. Castin, and C. Salomon, Science 296, 1290 (2002); K. E. Strecker, G. B. Partridge, A. G. Truscott, and R. G. Hulet, Nature (London) 417, 150 (2002); U. Al Khawaja, H. T. C. Stoof, R. G. Hulet, K. E. Strecker, and G. B. Partridge, Phys. Rev. Lett. 89, 200404 (2002); S. K. Adhikari, New J. Phys. 5, 137 (2003).

[3] E. A. Donley, N. R. Claussen, S. L. Cornish, J. L. Roberts, E. A. Cornell, and C. E. Wieman, Nature (London) 412, 295 (2001).

[4] J. L. Roberts, N. R. Claussen, S. L. Cornish, E. A. Donley, E. A. Cornell, and C. E. Wieman, Phys. Rev. Lett. 86, 4211 (2001); S. L. Cornish, N. R. Claussen, J. L. Roberts, E. A. Cornell, and C. E. Wieman, ibid. 85, 1795 (2000).

[5] S. Inouye, M. R. Andrews, J. Stenger, H. J. Miesner, D. M. Stamper-Kurn, and W. Ketterle, Nature (London) 392, 151 (1998); Ph. Courteille, R. S. Freeland, D. J. Heinzen, F. A. van Abeelen, and B. J. Verhaar, Phys. Rev. Lett. 81, 69 (1998).

[6] H. Saito and M. Ueda, Phys. Rev. Lett. 86, 1406 (2001); Y. Kagan, A. E. Muryshev, and G. V. Shlyapnikov, ibid. 81, 933 (1998). R. A. Duine and H. T. C. Stoof, ibid. 86, 2204 (2001).

[7] S. K. Adhikari, Phys. Rev. A 66, 013611 (2002).
[8] S. K. Adhikari, Phys. Rev. A 66, 043601 (2002); Phys. Lett. A 296, 145 (2002).

[9] H. Saito and M. Ueda, Phys. Rev. A 65, 033624 (2002).

[10] M. Ueda M and H. Saito, J. Phys. Soc. Jpn. 72, 127 (2003).

[11] L. Santos and G. V. Shlyapnikov, Phys. Rev. A 66, 011602(R) (2002).

[12] C. M. Savage, N. P. Robins, and J. J. Hope, Phys. Rev. A 67, 014304 (2003).

[13] R. A. Duine and H. T. C. Stoof, Phys. Rev. A 68, 013602 (2003).

[14] E. A. Calzetta and B. L. Hu, Phys. Rev. A 68, 043625 (2003).

[15] W. Bao, D. Jaksch, and P. A. Markowich, J. Phys. B 37, 329 (2004).

[16] S. Wüster, J. J. Hope, and C. M. Savage, Phys. Rev. A 71, 033604 (2005)

[17] S. K. Adhikari, J. Phys. B 37, 1185 (2004).

[18] S. K. Adhikari, Phys. Rev. A 70, 043617 (2004).

[19] F. Dalfovo, S. Giorgini, L. P. Pitaevskii, and S. Stringari, Rev. Mod. Phys. 71, 463 (1999).

[20] V. S. Shchesnovich, A. M. Kamchatnov, and R. A. Kraenkel, Phys. Rev. A 69, 033601 (2004); S. K. Adhikari, ibid. 63, 043611 (2001); V. S. Shchesnovich and S. B. Cavalcanti, ibid. 71, 023607 (2005).

[21] G. Modugno, G. Roati, F. Riboli, F. Ferlaino, R. J. Brecha, and M. Inguscio, Science 297, 2240 (2002).

[22] F. S. Cataliotti, S. Burger, C. Fort, P. Maddaloni, F. Minardi, A. Trombettoni, A. Smerzi, and M. Inguscio, Science 293, 843 
(2001); S. K. Adhikari, Eur. Phys. J. D 25, 161 (2003).

[23] F. S. Cataliotti, L. Fallani, F. Ferlaino, C. Fort, P. Maddaloni, and M. Inguscio, New J. Phys. 5, 71 (2003); S. K. Adhikari, Nucl. Phys. A 737, 289 (2004).

[24] M. Greiner, O. Mandel, T. Esslinger, T. W. Hansch, and I. Bloch, Nature (London) 415, 39 (2002); S. K. Adhikari and P. Muruganandam, Phys. Lett. A 310, 229 (2003).

[25] O. Morsch, J. H. Müller, D. Ciampini, M. Cristiani, P. B. Blakie, C. J. Williams, P. S. Julienne, and E. Arimondo, Phys.
Rev. A 67, 031603(R) (2003); O. Morsch, M. Cristiani, J. H. Müller, D. Ciampini, and E. Arimondo, ibid. 66, 021601(R) (2002); Laser Phys. 13, 594 (2003); S. K. Adhikari, J. Phys. B 36, 3951 (2003).

[26] J. L. Roberts, N. R. Claussen, S. L. Cornish, and C. E. Wieman, Phys. Rev. Lett. 85, 728 (2000).

[27] S. K. Adhikari and P. Muruganandam, J. Phys. B 35, 2831 (2002); P. Muruganandam and S. K. Adhikari, ibid. 36, 2501 (2003). 